\title{
VIRGO: A WIDE BAND GRAVITATIONAL WAVE DETECTOR
}

B. Caron, A. Dominjon, C. Drezen, R. Flaminio, X. Grave, F. Marion,

L. Massonnet, C. Mehmel, R. Morand, B. Mours, V. Sannibale, M. Yvert

LAPP, Annecy-Le-Vieux, France

D. Babusci, S. Bellucci, G. Candusso, G. Giordano, G. Matone

INFN Frascati, Frascati, Italy

L. Dognin, J. M. Mackowski, M. Napolitano, L. Pinard

IPN Lyon, Villeurbanne, France

F. Barone, E. Calloni, L. Di Fiore, A. Grado, L. Milano, G. Russo, S. Solimeno

INFN Napoli, Napoli, Italy

M. Barsuglia, V. Brisson, F. Cavalier, M. Davier, P. Hello, F. LeDiberder, P. Marin, M. Taubman $L A L$, Orsay, France

F. Bondu, A. Brillet, F. Cleva, H. Heitmann, L. Latrach, C.N. Man, Pham-Tu Manh, J.-Y. Vinet VIRGO laser group, Orsay, France

C. Boccara, Ph. Gleyzes, V. Loriette, J.P. Roger

ESPCI, Paris, France

G. Cagnoli, L. Gammaitoni, J. Kovalik, F. Marchesoni, M. Punturo

INFN Perugia, Perugia, Italy

M. Bernardini, S. Braccini, C. Bradaschia, R. Del Fabbro, R. DeSalvo, A. Di Virgilio, I. Ferrante,

F. Fidecaro, A. Gennai, A. Giassi, A. Giazotto, L. Holloway, P. La Penna, G. Losurdo,

F. Palla, Pan Hui-Bao, A. Pasqualetti, D. Passuello, R. Poggiani, G. Torelli, Zhang Zhou INFN Pisa, Pisa, Italy

E. Majorana, P. Puppo, P. Rapagnani, F. Ricci

INFN Roma, Roma, Italy

presented by

RAFFAELE FLAMINIO

at

Mathematical Aspects of Theories of Gravitation

Warsaw, Poland, March 1996

1. Introduction. VIRGO $[1,2]$ is a collaborative effort between the CNRS in France and the INFN in Italy. It involves scientists from Frascati, Napoli, Perugia, Pisa and

1991 Mathematics Subject Classification: 83C35, 83B05.

The paper is in final form and no version of it will be published elsewhere. 
Rome in Italy and from Annecy, Lyon, Orsay and Paris in France. The goal of the project is the construction of a Michelson interferometer of $3 \mathrm{~km}$ arm length, aimed at detecting gravitational waves in the frequency range from $10 \mathrm{~Hz}$ to a few $\mathrm{kHz}$. The detector, currently under construction, is located in Cascina, Italy, $10 \mathrm{~km}$ from Pisa.

The radiation of gravitational waves from accelerating masses is predicted by general relativity. According to the theory, gravitational waves propagate at the speed of light and they are transverse to the propagation direction. As for electromagnetic waves, there are two independent polarisation states, but the wave force field has a quadrupolar symmetry and the transformation property under rotation are those of a spin 2 wave (see figure 1).

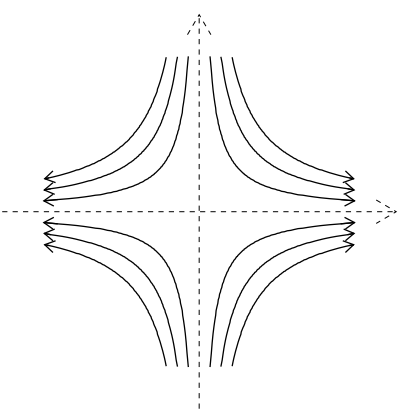

polarisation +

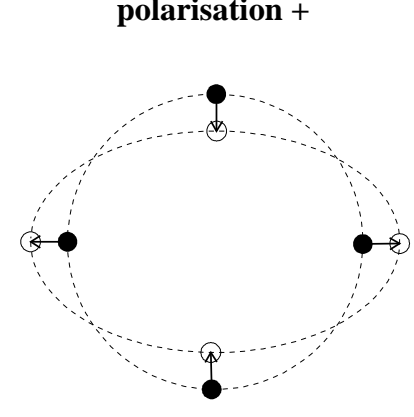

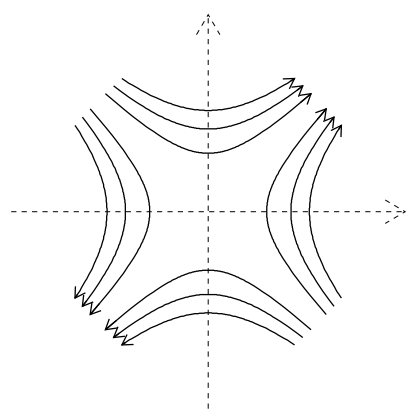

polarisation $\times$

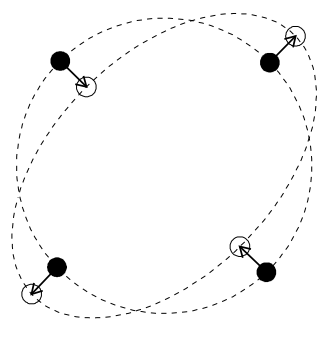

Figure 1: Gravitational wave force field (above) and the effect on a set of masses placed on a circle (below).

The small coupling constant makes the generation of gravitational waves on earth impossible at present. On the other hand many astrophysical process are believed to be sources of gravitational waves [3]. Processes involving compact objects in fast motion like coalescing binaries, supernovae explosions and rotating neutron stars are some of the astrophysical phenomena that should produce gravitational waves. The period slow down of the binary pulsar PSR 1913+16 [4] confirms the theoretical prediction and represents the first indirect observation of a gravitational wave emission process. 
The wave force field causes an antisymmetric change of the distance between free masses placed along two orthogonal directions transverse to the propagation direction of the wave (see figure 1). As a gravitational wave propagates across a Michelson interferometer having free suspended masses as mirrors, the interferometer arm lengths are changed antisymmetrically and a variation of the light power transmitted at the interferometer output port will be observed [5]. Interferometric detectors like VIRGO are based on this principle.

The difference in the displacements along two orthogonal directions $\delta \mathrm{L} / \mathrm{L}=\mathrm{h}$ is a measurement of the gravitational wave amplitude. A process involving the conversion of 0.01 solar masses into gravitational waves at the distance of the VIRGO cluster (10 Mpc) would produce a gravitational wave with an amplitude of about $\mathrm{h}=10^{-21}$ at the earth. Such a gravitational wave would cause a displacement of about $10^{-18} \mathrm{~m}$ between two free masses placed $1 \mathrm{~km}$ apart .

2. Detector overview. The VIRGO interferometer optical scheme is shown in figure 2. The change in length of the interferometer arms induced by a gravitational wave is proportional to the length of the arms. As a consequence, the sensitivity of such a detector increases linearly with such lengths. Practical problems and cost limit the length of such a detector to a few $\mathrm{km}$. The signal is increased by placing a Fabry-Perot in each arm of the interferometer to bounce the light back and forth along the arms many times. Thus the optical path can be increased up to more than $100 \mathrm{~km}$.

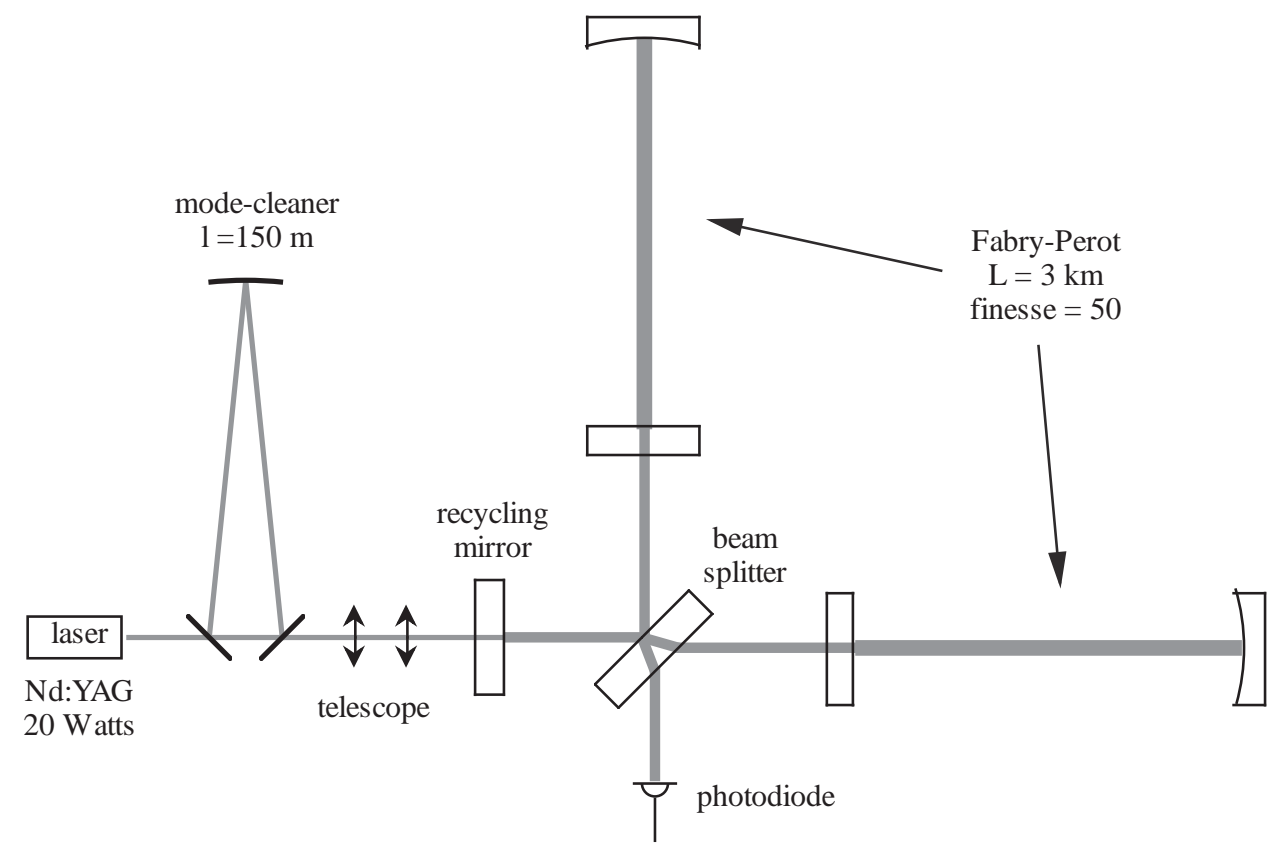

Figure 2: VIRGO: interferometer optical scheme. 
One of the main limitations to the sensitivity of a laser interferometer gravitational wave detector is the photon shot-noise. In order to reduce the effect of this noise the light power impinging on the beam splitter should be as high as possible. Current technology allows to build lasers delivering a power of the order of tens of Watts with the required frequency and power stability. By adding an additional mirror in between the laser and beam splitter it is possible to "recycle" the unused light reflected towards the laser thus increasing the light power impinging on the beam splitter. This technique, called power, recycling [6] is equivalent to using a more powerful laser.

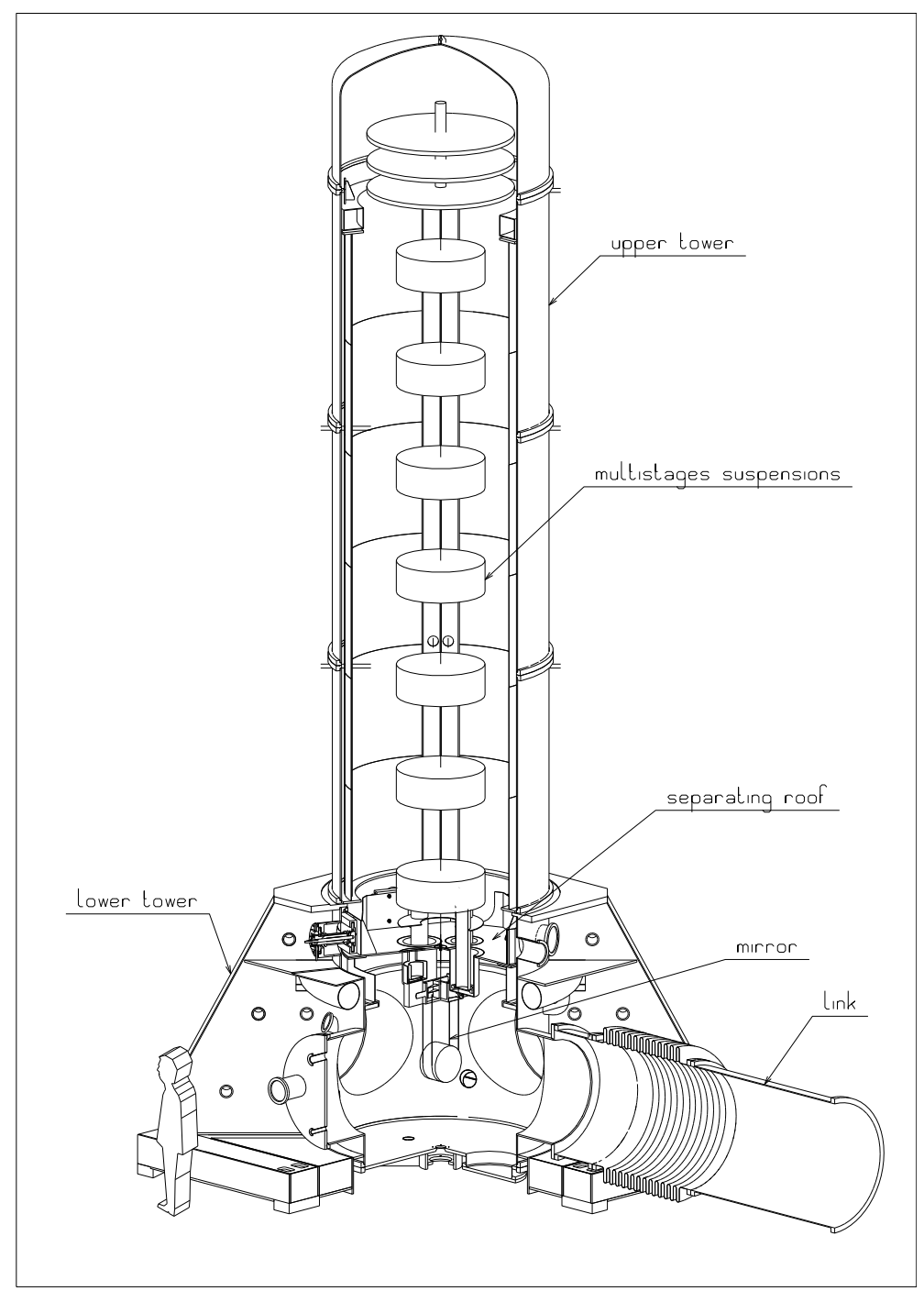

Figure 3: A view of the seismic isolation system inside the vacuum tank. 
The VIRGO collaboration will use these techniques in order to reach a power of more than $10 \mathrm{~kW}$ inside the Fabry-Perot cavities. This should allow to reduce the shot noise level to an equivalent strain spectrum noise of $310^{-23} / \sqrt{\mathrm{Hz}}$ at $100 \mathrm{~Hz}$. The required light source stability will be obtained using a stabilised injection locked Nd:YAG laser delivering 20 Watts.

In order to increase the amount of recycled light, the losses due to scattering and/or absorption inside the mirrors and on the reflecting coating layers should be kept as small as possible. All the optics should respect very stringent specifications in order to keep losses down to a few ppm. This is especially true for the Fabry-Perot mirrors. Furthermore these mirrors have to be relatively large in order to deal with the beam dimensions $(10 \mathrm{~cm}$ diameter at the end mirror) and quite massive in order to reduce the effect of pendulum thermal noise. The VIRGO interferometer will use as mirrors cylinders of fused silica 35 $\mathrm{cm}$ in diameter and 10 or $20 \mathrm{~cm}$ thick. The mirrors surfaces will be polished in their central area to a precision of $\lambda / 100$. The coating of all large optics is developed in the VIRGO experiment by the Lyon group.

In order to detect the tiny displacement induced by the expected gravitational waves the mirror should be completely isolated from all external perturbations. The effect of seismic noise will be reduced by suspending all the mirrors to a cascade of seven pendulum stages each $1 \mathrm{~m}$ in length, called Super-Attenuator [7] (see figure 3). Vertical compliance is added to each stage by means of pre-stressed metal cantilever blades whose spring constant is softened with magnetic anti-springs. Thus each stage has resonant frequencies below $1 \mathrm{~Hz}$ in all six degrees of freedom. This system will provide an attenuation of the seismic vibration by more than ten orders of magnitude at $10 \mathrm{~Hz}$.

All the optical cavities will be kept at resonance by a complex system of servos using the signals read by several photodiodes and acting on the mirrors position. These servo will also keep the interferometer output on the null interference condition in order to maximise the detector sensitivity. Other independent control loops will act on the suspension in order to reduce the low frequency oscillation amplitude and on the laser in order to keep the frequency and the power at the required level of stability.

Acoustic noise as well as air index of refraction fluctuations may also limit the sensitivity of such a detector. To avoid these effects the whole interferometer should be placed under vacuum. The VIRGO vacuum system will consist of two $3 \mathrm{~km}$ long and $1.2 \mathrm{~m}$ diameter tubes and several $10 \mathrm{~m}$ high towers containing the required suspensions. Along each tube eleven pumping stations will keep the pressure level below $10^{-9}$ mbar.

3. Detector sensitivity. The VIRGO expected sensitivity expressed in terms of an equivalent strain spectrum is shown in figure 4.

The use of the Super-Attenuator should keep the seismic noise effects negligible above a few hertz. From a few $\mathrm{Hz}$ up to $100 \mathrm{~Hz}$ the sensitivity will be limited by pendulum thermal noise. The effect of the pendulum thermal noise decreases as the frequency increases and around $100 \mathrm{~Hz}$ the effect of mirror internal thermal noise is expected to be the main limit to the detector sensitivity. Around $200 \mathrm{~Hz}$ the sensitivity reaches the lowest level at about $310^{-23} / \sqrt{\mathrm{Hz}}$. 


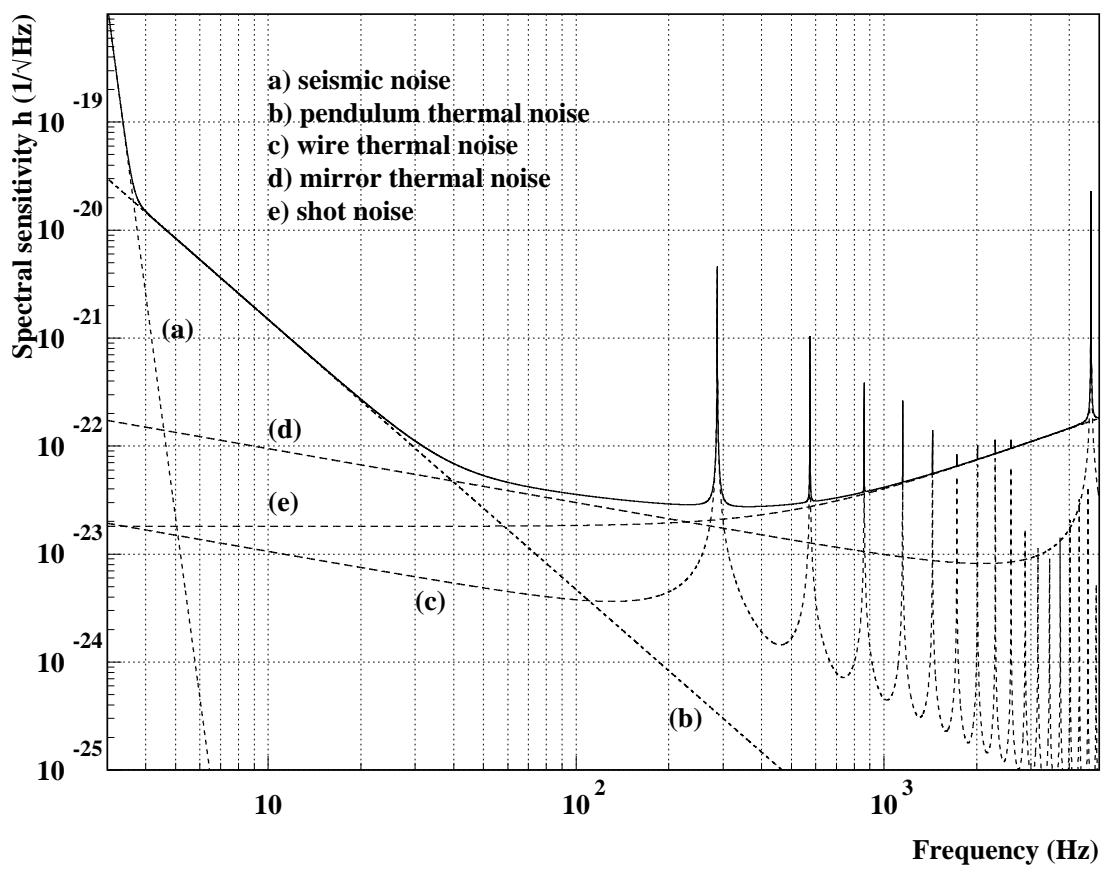

Figure 4: The expected VIRGO spectral sensitivity

Above a few hundred $\mathrm{Hz}$ the light storage time in the arms becomes non negligible and the interferometer response decreases. As a consequence the shot noise level gets larger and it becomes the main limit to the sensitivity.

Various resonances peaks are visible above $300 \mathrm{~Hz}$. These peaks in the noise spectrum are due to the excitation by thermal noise of the violin modes in the mirror suspension wires.

4. Status and schedule. The VIRGO experiment has been approved by INFN and CNRS in 1993.

Construction of the central building just started and it is expected to be completed in about one year. During 1998 the vacuum system, suspensions and laser will be installed in the central building. A short $6 \mathrm{~m}$ long interferometer using smaller mirrors will be installed in the central building in order to carry on test on the integration of the various systems.

In the meanwhile the construction of the $3 \mathrm{~km}$ long arms and of the terminal buildings will be completed and the tube will be installed. Installation of the large mirrors and first operation of the whole system is expected during the year 2000 .

\section{References}

[1] VIRGO Coll., Final Conceptual Design (1992).

[2] VIRGO Coll., Final Design, Version 0 (1995). 
[3] K. S. Thorne, Gravitational Radiation in: 300 Years of Gravitation, Cambridge University Press (ed.), Cambridge, (1987), 330-452.

[4] J. H. Taylor and J. M. Weisberg, The Astrophysical Journal 253, (1982), 908-920.

[5] M. E. Gersenshtein and V. I. Pustovoit, Sov. Phys. - JETP 16, (1963), 433

R. Weiss, Q.Prog. Rep. Lab. Electron. MIT 105, (1972), 54.

[6] R. W. P. Drever, Gravitational Radiation, les Houches 1982, eds N.Deruelle and T.Piran (North-Holland Amsterdam), (1983), 321.

[7] A. Giazotto, Physics Reports 182, (1989), 365-425. 